\title{
DESIGN AND PERFORMANCE ANALYSIS OF VCO FOR STANDARD GSM USING MEMS
}

\author{
Khalid A. S. Al-Khateeb* and WAJdi F. Al-Khateeb \\ Department of Electrical and Computer Engineering, \\ Kulliyah of Engineering, International Islamic University Malaysia, \\ P.O. Box 10, Kuala Lumpur, 50728, MALAYSIA \\ "E-mail:khalid@iiu.edu.my
}

\begin{abstract}
The design of a prototype monolithic Micro Electro-Mechanical Systems (MEMS) electronic circuits, namely the Voltage Controlled Oscillators (VCOs) is presented. The components can achieve the stringent requirements of wireless communication applications such as GSM cellular telephony. The VCO meets the low phase noise specifications of $-136 \mathrm{dBc} / \mathrm{Hz}$ at large offset frequency of $3 \mathrm{MHz}$, over the appropriate frequency range. The model of the monolithic VCO is based on the topology of the Colpitts Oscillator. It is relatively less complicated, which facilitates the practical integration of the MEMS components into the configuration. The variable capacitor and the monolithic 3-D coil inductor are suitable for low phase-noise and low power consumption at the application frequencies. A PSpice simulation model was developed with MEMS switching devices that can be integrated into the system. The model helps in determining the design parameters, which affect the performance and operation reliability of the RF transceiver system, for which a prototype has been tested and proved successful.
\end{abstract}

KEYWORDS : MEMS; Electronic Circuits; VCO; GSM; Phase Noise.

\section{INTRODUCTION}

Mobility and portability are features, which have a strong driving force in the miniaturization process of wireless communication interfaces. They have raised much interest in single-chip applications. The integration of passives devices such as variable capacitors and inductors, typically involves some trade-offs between noise, power consumption, linearity, frequency range, gain and supply voltage. Higher Q-factors and lower insertion losses however, may mitigate some of the trade-offs. A low phase noise VCO operating at radio frequency (RF) can be designed by integrating a variety of suitable MEMS components. Other applications that may involve MEMS capacitive switches are output-stage audio amplifiers, which should satisfy the GSM specifications. The important design parameters however, have to be identified before the implementation of such circuits. MEMS can be used to replace standard variable capacitors (varicaps) and inductors. Both of these passive components can be fabricated on silicon substrates and thus are amenable to monolithic integration by standard IC processes. The MEMS technology is attractive for the implementation of on-chip high-quality RF variable capacitors and inductors, especially in low noise power amplifiers, matching networks, 
and monolithic low-noise VCOs. In fact, the Q-factor of these components may ultimately determine the phase noise performance, which is critical in high performance communication systems [1]. The introduction of MEMS in RF systems increases the functionality and improves the performance. The challenge however remains in how to minimize the phase noise while maintaining lowest possible power consumption..

\section{THE PHASE NOISE}

The requirements of a versatile VCO are low phase noise, low power consumption, wide tuning range and a high power output. However, to achieve these characteristics all at once is a challenging task, because of trade-offs. Much of the research is aimed at reducing the phase noise, which is a rapid short term random fluctuation. It can be expressed in terms of signal to noise power ratio in unit bandwidth. The expression, which can be used to calculate the noise power at the offset frequency $f_{m}$, is based on the classical Leeson's model [2]:

$$
P N\left(f_{m}\right)=\frac{2 \cdot k \cdot T \cdot F \cdot R_{p}}{A_{o}{ }^{2}}\left(\frac{f_{o}}{2 \cdot Q \cdot f_{m}}\right)^{2}\left(1+\frac{\Delta f_{1 / f^{3}}}{f_{m}}\right)^{2} \ldots
$$

Where; $\boldsymbol{F}$ is the excess noise factor, $\boldsymbol{k}$ Boltzmann constant, $\boldsymbol{T}$ the absolute temperature, $\boldsymbol{A}_{\boldsymbol{o}}$ the amplitude of oscillation, $\boldsymbol{Q}$ the resonator loaded quality factor, $\boldsymbol{R}_{\boldsymbol{p}}$ is the parallel resistance to model losses in the resonator, and $\Delta \boldsymbol{f}_{1} / \boldsymbol{f}^{3}$ is the corner frequency in the phase noise spectrum.

For an ideal oscillator, the shape of the spectrum is an impulse at a resonant frequency, $\boldsymbol{\omega}_{\mathbf{0}}$. However, in actual oscillators, a skirt shape is formed around $\boldsymbol{\omega}_{\mathbf{0}}$. Figure 1(a) shows the single spike of energy at the center carrier frequency, $\boldsymbol{\omega}_{\mathbf{0}}$ and Figure 1(b) shows the skirts around $\omega_{0}$ due to phase noise. Actually, the phase noise is expressed as the power at particular offset, $\boldsymbol{\omega}_{\mathrm{m}}$ from $\boldsymbol{\omega}_{\mathbf{0}}$. The signal power is measured in a $\mathbf{1} \mathbf{H z}$ bandwidth at $\boldsymbol{\omega}_{\mathrm{m}}$ [3]. The unit of phase noise is $\mathbf{d B c} / \mathbf{H z}$ represented as decibels below the carrier per hertz.

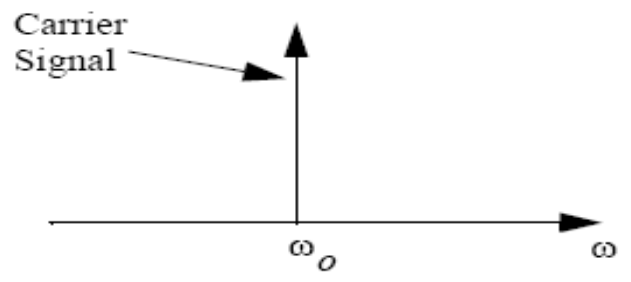

(a)

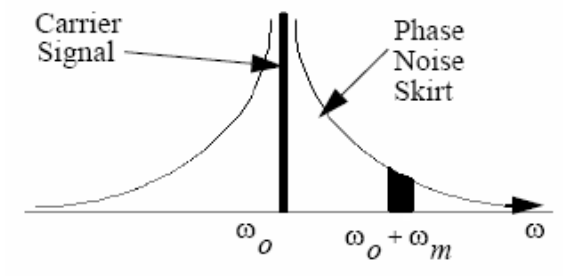

(b)

Fig. 1: (a) Output spectra of ideal oscillator, (b) Output spectra of practical noisy oscillator. 
In the prototype MEMS oscillator, there is an additional noise source due to the mechanical thermal vibration, which is included instead of thermal and flicker noise. In practice, this kind of additional noise is due to the vibrations of the suspended plate which cause variation in the capacitance and affect the overall output of the phase noise [1].

\section{MEMS PASSIVE DEVICES IN VCO}

There are two approaches to design and fabricate a VCO using MEMS passive devices. The first is to replace the MOS capacitors with MEMS, which is good for a wide tuning range. The second is to use a monolithic 3-D coil inductor. The high $\boldsymbol{Q}$ varicap is a key element in a low phase noise VCO. The MEMS varicap also enables a complete monolithic fabrication of RF VCO for on-chip IC compatible devices, with lower loss, larger tuning range and higher linearity [4]. The high- $\boldsymbol{Q}$ variable capacitor can be realized by a surface micro-machined all aluminum microstructure. The top and cross-section are shown in Fig. 2. It consists of $1 \mu \mathrm{m}$-thick aluminum plate suspended in air $1.5 \mu \mathrm{m}$ above the bottom layer and anchored with four folded beam suspensions acting as springs. Aluminum sheet resistance is low, which is critical to minimize the ohmic losses, and the fabrication temperature of $150^{\circ} \mathrm{C}$ [1] is also low. The plate size of $200 \mu \mathrm{m}$ by $200 \mu \mathrm{m}$ and $1.5 \mu \mathrm{m}$ nominal air gap results in a nominal capacitance value of approximately $200 \mathrm{pF}$. Thus, large capacitance can be obtained by parallel connection [5]. In RF transceivers, the DC tuning voltage is typically limited to $3.3 \mathrm{~V}$ or less. Thus with a $1.5 \mu \mathrm{m}$ air gap and a $200 \mu \mathrm{m}$ square plate it requires $3.8 \mathrm{~N} / \mathrm{m}$ for a $3.3 \mathrm{~V}$ operation. This corresponds to a mechanical resonant frequency of $30 \mathrm{kHz}$ [1]. Each of the suspension four folded beams is $100 \mu \mathrm{m}$ long and $20 \mu \mathrm{m}$ wide as in Fig. 2.
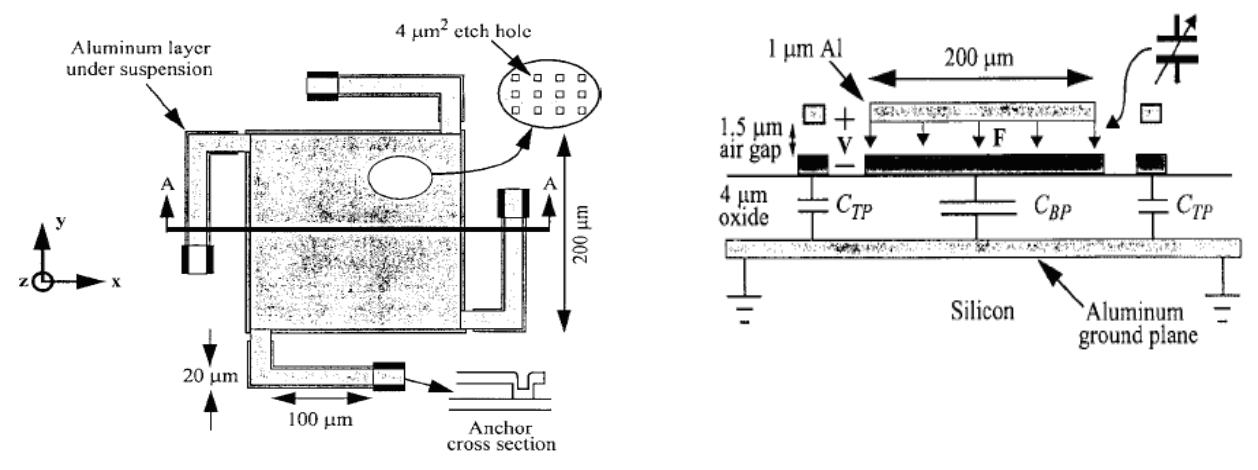

Fig. 2: Micro-machined variable capacitor.

A high $\boldsymbol{Q}$ inductor is another key element in low phase noise VCO, since the inductors produced by silicon processes cannot provide high $\boldsymbol{Q}$ factors, due to ohmic loss in the thin metal layers and eddy-currents [6]. Several methods have been proposed. However, the bond-wires types are chosen as an attractive alternative because the $\boldsymbol{Q}$ values are at least an order of magnitude higher than the on-chip spiral inductors. They have been 
implemented in RF VCOs and high efficiency power amplifiers. The 3-D microstructure also minimizes the capacitive coupling and eddy current loss, leading to larger $\boldsymbol{Q}$-factor at higher frequencies. Bigger inductors and smaller capacitors allow operation with less power [6].

In a high $\boldsymbol{Q}$ inductor every mm of bond-wire contributes about $1 \mathrm{nH}$. Therefore, a few $\mathrm{nH}$ requirement for wireless applications can be provided readily, with a lead frame of a standard IC package [1].
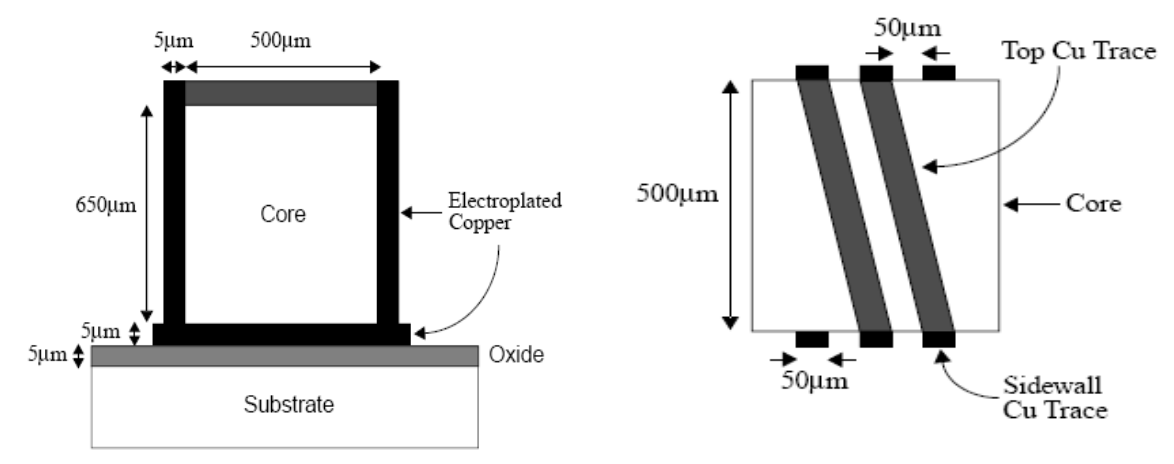

Fig. 3: 3-D coil inductor.

The 3-D coil inductor, shown in Fig. 3, consists of two $5 \mu \mathrm{m}$ thick turns, $50 \mu \mathrm{m}$ wide copper traces electroplated around an insulating core with a $650 \mu \mathrm{m}$ by $500 \mu \mathrm{m}$ crosssection. The core is alumina, which has negligible loss tangent, which is another key parameter to ensure high $\boldsymbol{Q}$. A core width of $500 \mu \mathrm{m}$ is found experimentally to be the minimum that avoids tilting during attachment. The fabrication process is described in details elsewhere [8].

\section{THE DESIGN PROCEDURE OF THE VCO}

There are many types of oscillator topologies which can be used to construct an RF $\mathrm{VCO}$ for frequencies below $1 \mathrm{MHz}$. For frequencies above $1 \mathrm{MHz}$ however, LC feedback oscillators are normally used. Due to the frequency limitations of most op-amps, transistor amplifiers are used as the gain elements. Thus, a popular topology, which proved successful in many commercial modules, is the Colpitts oscillator (Fig. 4), due to its simplicity, robustness and wide range of operating frequencies, from IF to RF [8].

The frequency is determined by the resonant frequency of the feedback network, which is a parallel resonant tank circuit, in which any change in the inductor or capacitor will change the oscillation frequency, $\boldsymbol{f}_{\boldsymbol{o}}$ given by:

$$
f_{o}=\frac{1}{2 \pi \sqrt{L C_{T}}}
$$


where, $\mathbf{C}_{\mathbf{T}}=\mathbf{C}_{\mathrm{v}}+\mathbf{C}_{12}, \mathbf{C}_{\mathbf{v}}=\left(\mathbf{C}_{\mathrm{var}} \times \mathbf{C}_{\mathbf{0}}\right) /\left(\mathbf{C}_{\mathrm{var}}+\mathbf{C}_{\mathbf{0}}\right)$, and $\mathbf{C}_{12}=\left(\mathbf{C}_{1} \times \mathbf{C}_{2}\right) /\left(\mathbf{C}_{1}+\mathbf{C}_{2}\right)$

(Note: The above equation is accurate only if the LC circuit has a high $\boldsymbol{Q}$ ).

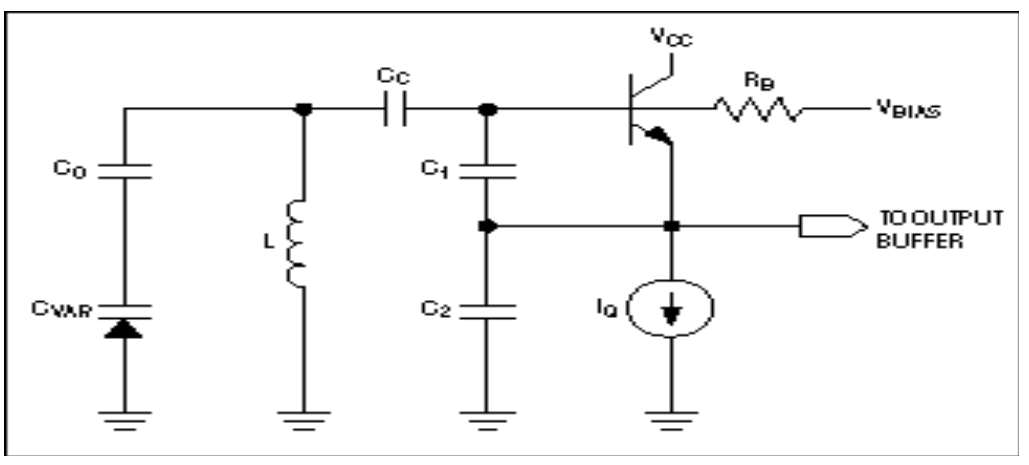

Fig. 4: Colpitts circuit topology for VCO.

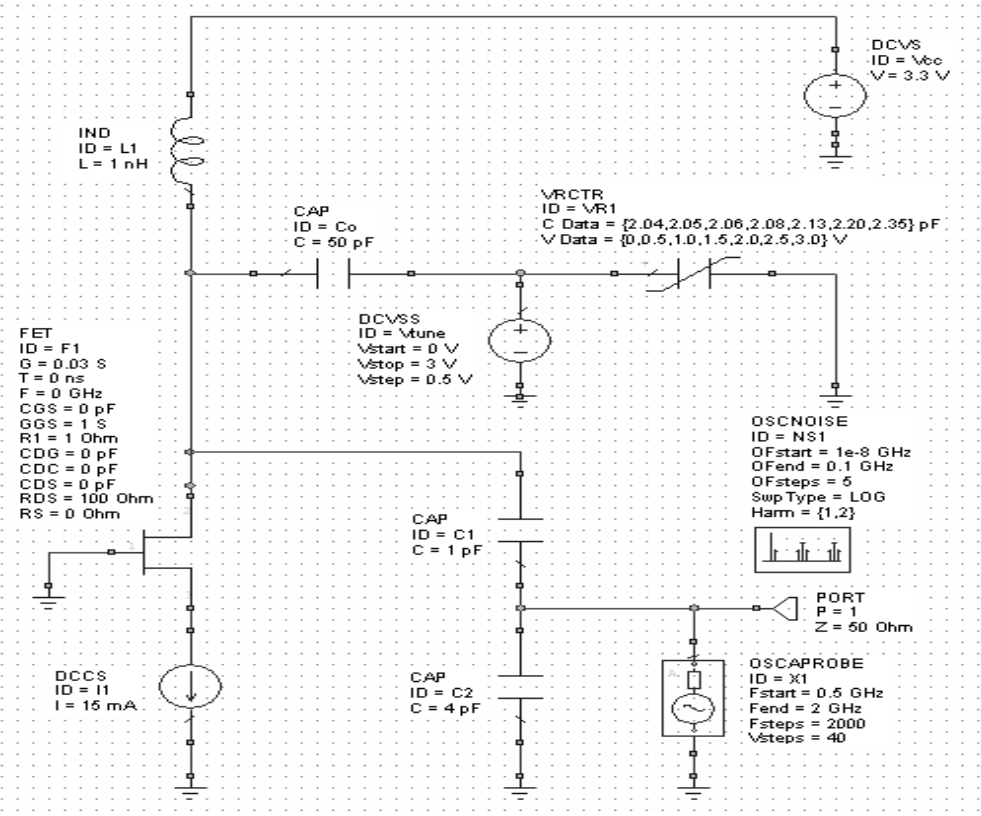

Fig. 5: The actual circuit design of the VCO.

Considering the Colpitts VCO (Fig. 5), which uses parallel-mode LC tank configuration, the analysis is straightforward. The FET is a medium power amplifier as compared to a bipolar transistor. It may provide double the power for a given frequency or double frequency for a given power. In fact, FET can be used up to $30 \mathrm{GHz}$. At $10 \mathrm{GHz}$, the FET itself may actually provide several watts of output power [9]. In the simulation model however, the VCO operates at a frequency $\boldsymbol{f}_{\boldsymbol{o}}$ of approximately $1 \mathrm{GHz}$. 
The task for phase noise performance analysis is to reconfigure the Colpitts oscillator as an amplifier with positive LC feedback, as in Fig. 5. Hence a readily available set of design equations can be used, which are clear and convenient. Instead of only analyzing the phase noise, this model can also be useful in calculating the loop gain and the amplitude of the oscillation [9].

The factors that affect the accuracy of phase noise simulation and measurement are many. It may be possible addressed these factors accurately with a prudent selection of the passive components and proper resonator modeling. For an acceptable level of simulation accuracy of a VCO that operates at RF and above, all components in the linear network such as transmission lines and discontinuities must be accurately characterized over several harmonics of the fundamental oscillation frequency. This is important because the accuracy of the oscillation signal affects the noise analysis, and the noise analysis itself depends on the linear network. This means that any inaccuracy in the network characterization will affect the quality of the phase noise simulation. The simulation accuracy of the VCO will ultimately determine the parameters of all the fabricated parts, including the dimensions of the circuit board, material properties, and component models of any parasitic behavior [10].

\section{ANALYSIS OF THE VCO DESIGN}

Microwave Office is a software, which can be useful in simulating many circuit designs. Therefore it has been for the simulation of the VCO. It also enables the prediction of the performance characteristics. The analysis can either be performed in the frequency domain using the harmonic balance technique, or in the time domain using transient simulators such as SPICE and Spectre. The simulation method involves three main steps. First, it attempts to locate the start-up frequency with respect to the well-known loop gain criterion. When the loop gain saturates to a magnitude of 1 in the large-signal steady state, the simulator will step the probe voltage in an attempt to detect loop gain saturation. Then, the neighborhood of gain saturation is used as the starting point for the analysis. Then the voltage and frequency of the probe are adjusted in a way that results in zero probe-current. After setting all the requirements, as in Fig. 6, the simulation begins to perform the analysis automatically.

The analysis uses a special device called oscillator probe that allows fast and robust oscillator simulations, even for the case of extremely high resonator $\boldsymbol{Q}$. The oscillator probe refers to the combination of the source and the ideal impedance element. In the Microwave Office simulator, the oscillator probe is denoted as OSCAPROBE, as shown in Fig. 7. The most significant probe parameters are Fstart and Fend. These parameters indicate the range of search for start-up frequency. Another parameter is the Fsteps, which refers to the number of steps used in the search for start-up frequency and rarely needs to be changed from default. However, in extremely high- $\boldsymbol{Q}$ cases, Fsteps may be increased or the frequency range is narrowed. The recommended location of the oscillation probe is at a node connecting the resonator and the active device. 


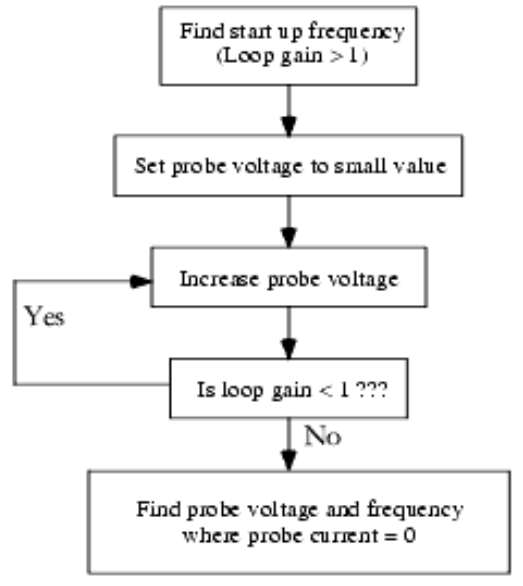

Fig. 6: Flow chart of the analysis.

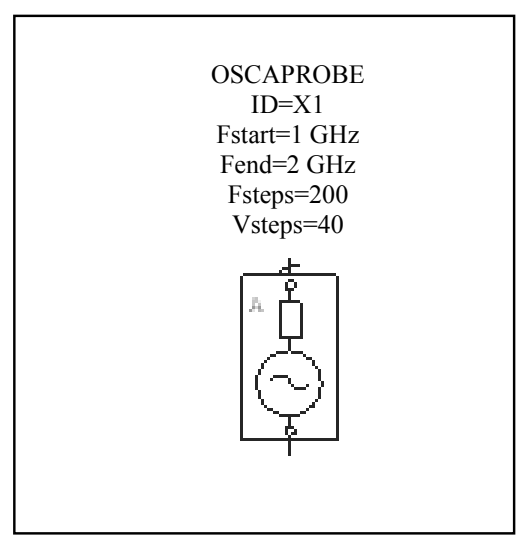

Fig. 7: Schematic symbol of $O S C A P R O B E$.

The main objective of simulating the VCO is to measure the phase noise performance, then to compare it with the values of a real prototype VCO at the offset frequency of $3 \mathrm{MHz}$. The OSCNOISE simulator shown in Fig 8 was employed to determine the phase noise performance of the VCO. The results are shown in Fig. 9. The phase noise is measured in $\mathrm{dB} / \mathrm{Hz}$ offset from the carrier and is plotted on a log frequency scale. In this simulation, the OFstart and OFend parameters were set to $1 \mathrm{e}-008 \mathrm{GHz}$ and $0.1 \mathrm{GHz}$ respectively.

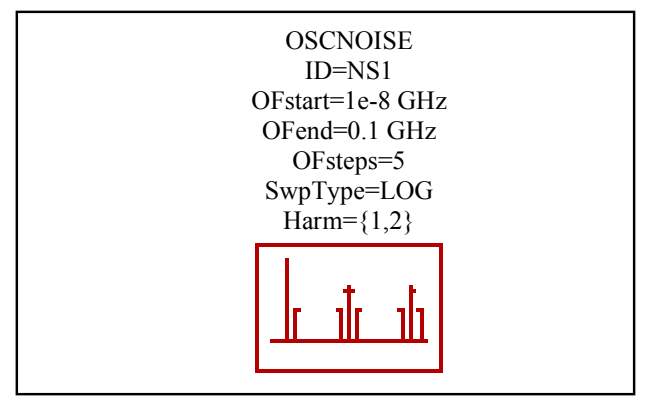

Fig. 8: Schematic symbol of OSCNOISE.

It is shown that at an offset frequency of approximately $3 \mathbf{M H z}$, the value of the phase noise is $\mathbf{- 1 3 8 . 0 5} \mathbf{~ d B c / H z}$, which is in good agreement with the actual value for the prototype $\mathbf{- 1 3 6} \mathbf{~ d B c / H z}$. The noise components in conventional LC tuned oscillators are; electrical thermal noise, flicker noise ( $/ / \boldsymbol{f}$ noise), supply voltage noise, and the noise contribution from the substrate. In practical MEMS tuned LC oscillators however, additional phase noise is introduced by the mechanical thermal vibration of the variable capacitors. The vibration of the suspended plates causes variation in the capacitance value, which results in phase noise or jitter in the output frequency [1]. The noise power spectral density due to plate displacement can be expressed as follows: 


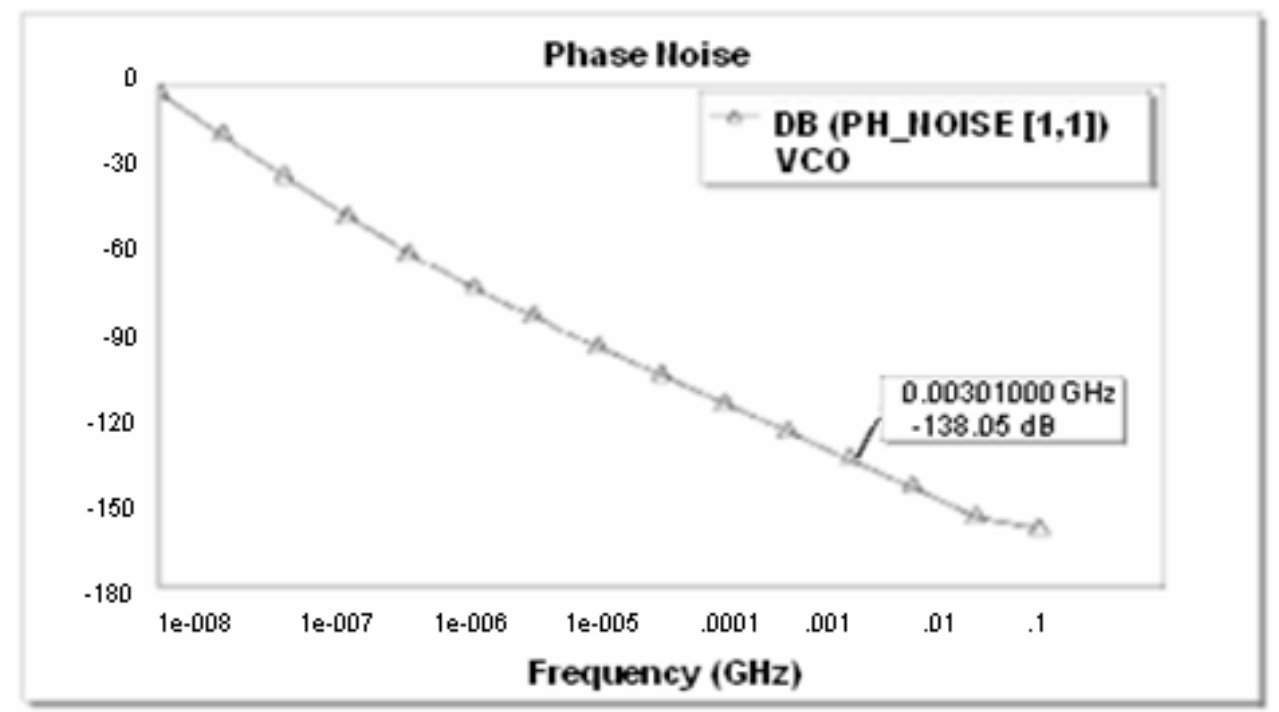

Fig. 9: Plot of the simulated phase noise.

$$
\overline{X_{n}^{2}(\omega)}=\frac{4 \cdot k \cdot T \cdot b}{k_{m}^{2}\left[\left(1-\frac{\omega^{2}}{\omega_{n}^{2}}\right)^{2}+\frac{1}{Q_{M}^{2}} \frac{\omega^{2}}{\omega_{n}^{2}}\right]}
$$

Where $\boldsymbol{k}$ is Boltzmann constant, $\boldsymbol{T}$ is the absolute temperature, $\boldsymbol{b}$ is damping coefficient due to the surrounding gas ambient and internal dissipation of the system, $\boldsymbol{\omega}_{\boldsymbol{n}}$ is the mechanical resonant frequency of the capacitor, $\boldsymbol{k}_{\boldsymbol{m}}$ is the structure compliance and $\boldsymbol{Q}_{\boldsymbol{M}}$ is the mechanical quality factor. Additional phase noise can be further expressed as given below:

$$
S_{\theta}\left(f_{m}\right)_{\text {Brownian }}=\frac{\overline{X_{n}^{2}\left(f_{m}\right)}}{8\left(\frac{1+\alpha}{\alpha}\right)^{2} N x_{o}^{2}}\left(\frac{f_{0}}{f_{m}}\right)^{2}
$$

Where $\boldsymbol{x}_{\boldsymbol{o}}$ is the nominal air gap of the capacitor, $\boldsymbol{N}$ is the number of parallel-connected devices and $\boldsymbol{\alpha}$ is the ratio between the nominal tank tunable capacitance and its parasitics, $\boldsymbol{f}_{\boldsymbol{o}}$ and $\boldsymbol{f} \boldsymbol{m}$ are the oscillation and the offset frequencies, respectively.

These two equations enable the phase noise induced by the Brownian motion to be determined at various offset frequencies. For a typical design condition in which $\boldsymbol{x}_{\boldsymbol{o}}=$ $1.5 \mu \mathrm{m}, Q_{M} \cong 1$ at $1 \mathrm{~atm}, \omega_{n}=2 \pi(30 \mathrm{kHz}), N=4, \alpha \cong 0.5$, and $f_{o}=1 \mathrm{GHz}$, the phase noise at offset frequencies $f_{m}$ of $10 \mathrm{KHz}, 100 \mathrm{KHz}$, and $3 \mathrm{MHz}$ are respectively [1] $64 \mathrm{dBc} / \mathrm{Hz},-105 \mathrm{dBc} / \mathrm{Hz}$, and $-136 \mathrm{dBc} / \mathrm{Hz}$, Typical wireless communication applications specify low phase noise requirement at relatively large offset frequency, for example $\mathbf{- 1 3 6}$ 
$\boldsymbol{d B c} / \mathbf{H z}$ at $3 \mathbf{M H z}$ offset for $\mathbf{G S M}$. In order to fulfill these norms, the VCO phase noise must be maintained better than $-135 \mathrm{dBc} / \mathbf{H z}$ at $3 \mathbf{M H z}$ offset over the appropriate frequency range of $855 \mathrm{MHz}$ to $863 \mathrm{MHz}$.

\section{CONCLUSION}

MEMS tunable VCO is a viable proposition for GSM applications. It can provide superior performance characteristics over other types of VCO. It is expected to make large impact in many applications, especially in wireless mobile telephony. The phase noise analysis performed by simulation and described explicitly in this work is one of the most important aspects of VCO design. The Microwave Office software tool can be very useful in designing such circuits and predicting their performance. It is a powerful tool for simulation and design analysis of linear and nonlinear circuits and for measurements carried out under specifically set conditions. The practical application of the VCO simulation model was the fabrication using real MEMS passive devices i.e. the high-Q variable capacitors and 3-D coil inductors. The prototype in which MEMS constitute the frequency determining components of low phase-noise with low power consumption may prove to be commercially attractive, as it meets the stringent performance requirements of GSM at the application frequencies.

\section{REFERENCES}

[1] D. J. Young, V. Malba, A. F. Bemhardt, and B. E. Boser, "A Micromachined RF Low Phase Noise Voltage-Controlled Oscillator for Wireless Communications", IEDM Dig. Tech. Papers, pp. 285-300, 24 April 2001.

[2] D.B. Lesson, "A Simple Model of Feedback Oscillator Noise Spectrum", Proc. IEEE, 54 (2), pp. 329-330, 1996.

[3] V. K. Saraf, "Low Power Wide Tuning Range LC-VCO using RF MEMS Passives", Master thesis, Univ. of Carnegie Mellon, Pittsburgh, PA, August 2004.

[4] B. S. Darade and T. A. Parmar, "Low Phase Noise Fully Integrated VCO", 18th Intl. Conference on VLSI Design, Jan 2005.

[5] D. J. Young and B. E. Boser, "A Micromachined Variable Capacitor for Monolithic Low-Noise VCOS", Solid-State Sensor and Actuator Workshop, Dig. Tech. Papers, pp. 86-89, June 1996.

[6] E. C. Park, Y. S. Choi, J. Yoon, S. Hong and E. Yoon, "Fully Integrated Low PhaseNoise VCOs With On-Chip MEMS Inductors", IEEE Transactions on Microwave Theory and Techniques, vol. 51, no. 1, January 2003.

[7] D. J. Young, V. Malba, J. J. Ou, A. F. Bemhardt and B. E. Baser, "Monolithic HighPerformance Three-Dimensional Coil Inductors for Wireless Communication Applications", IEDM Dig. Tech. Papers, pp. 67-70, December 1997.

[8] R. H. Berube, "Computer Simulated Experiments for Electronic Devices Using Electronic Workbench", Prentice-Hall, Inc., 2000.

[9] C.O'Connor, "Develop A Trimless Voltage-Controlled Oscillator", Microwaves \& RF Magazine, pp. 94-105, 2000.

[10] D.Vye, "Improving VCO Phase Noise Performance Through Enhanced Characterization", High Frequency Electronics Magazine, pp. 56-60, 2004. 